\title{
Influences of a yoga intervention on the postural skills of the Italian short track speed skating team
}

This article was published in the following Dove Press journal:

Open Access Journal of Sports Medicine

12 February 2015

Number of times this article has been viewed

\author{
Jean-François Brunelle ${ }^{1,2}$ \\ Sébastien Blais-Coutu' \\ Kenan Gouadec ${ }^{3}$ \\ Éric Bédard ${ }^{3}$ \\ Philippe Fait ${ }^{1,2}$
}

'Department of Human Kinetics Sciences, ${ }^{2}$ Research Group on Neuromusculoskeletal Dysfunctions, Université du Québec à Trois-Rivières, QC, Canada; ${ }^{3}$ Italian Ice Sports Federation, Rome, Italy
Correspondence: Philippe Fait Université du Québec à Trois-Rivières, 335 I Boulevard des Forges, TroisRivières, QC G9A 5H7, Canada

$\mathrm{Tel}+$ I 819376501 I ext 3768

Fax +I 8193765092

Email philippe.fait@uqtr.ca
Introduction: In preparation for a short track speed skating season, eight men and seven women were given yoga sessions during an 8-week high volume training cycle. The sessions were planned according to the postural aspects specific to short track speed skating technical requirements. Three specific goals were selected for the intervention: 1) to observe whether the practice of yoga as postural training could improve the efficiency and the athlete's repertoire along the muscular synergies solicited in the short track speed skating specific technique; 2) to enhance and diversify the motor time-on-task of athletes without changing the prescription of other training stimulus; and 3) to lower the risk of injury during periods with high volumes of training.

Methods: A total of 36 sessions of yoga were given. Three postural tests were administered before and after the intervention with 14 angles analyzed. Non-parametric Wilcoxon test was used to compare angles' variations.

Results: The 36 yoga sessions totalized 986 minutes of motor time-on-task, registering a proportion of $30 \%$ of the global motor time-on-task of the training cycle. Improvements were found in eleven of the 14 angles measured when comparing pre- and post-postural tests ( $P$-value from 0.01 to 0.005$)$. During the 8 weeks, excepting traumatic injuries due to short track speed skating accidents, no skaters suffered injuries linked to the high volume of training. Following the intervention, coaches noticed, following their on-ice feedbacks, an adjustment in the efficiency of the skating technique, in particular regarding hip dissociation.

Conclusion: These results suggest that yoga could be inserted into out-of-season training cycles, even in a high volume training cycle. Planned with the decision training tools, it allows athletes to diversify their motor time-on-task by integrating a new functional range of generic movements with the solicitation of neuromuscular synergies related to the specificity of their sport.

Keywords: conditioning, motor time-on-task, physical literacy, athletic development, decision training

\section{Introduction}

\section{Context of the intervention}

In the modern era of professional and Olympic sports, every sport has a strong market culture. To achieve the highest levels, athletes, coaches, and other leaders must be rigorous and demonstrate their foresight in their way of trying to meet the demands of the market in which they find themselves. Therefore, the overall performance of an athlete or of a group of athletes requires the interaction of several factors presenting first, within each specific sports market, a dichotomy: what emanates from the body and the capacities of the athlete, the endogenous factors, and what comes from 
outside to influence and stimulate the athlete, the exogenous factors. Then, the technical factors, each time along with the internal logic of the market, are also influencing the nature of the motor skills the athletes are required to perform. As this article stages the Italian short track speed skating team, Table 1 lists the technical factors both endogenous and exogenous which influence a high level athlete evolving within the short track speed skating world cup circuit. ${ }^{1}$

Thus, we can resume by saying that short track speed skating requires the combination of having a proper end efficient skating technique, power in the lower limbs, stamina, and a strategy repertoire in a race situation. To bring their athletes to their top performance, coaches should first position themselves regarding influencing factors and will then be able to understand the many interactions, such as illustrated in Figure 1. ${ }^{1}$

Motor skills are split into three categories: locomotor activities, manipulation activities, and postural activities. ${ }^{2}$
Short track speed skating is mainly a locomotor activity but the efficiency of this locomotor action requires a specific posture, and because of its technical requirements, the basic position of speed skating, being itself a global muscle synergy, demands significant postural adjustments. In order to have an efficient skating technique, the athlete must take into account the positioning of his trunk tilted forward, the positioning of his hips, of his shoulders as well as the efficiency of his leg's extension and balance during both the push and recovery movements, as well as the cross-over and the pivot in the turns. ${ }^{3,4}$

The present article depicts 8 weeks of summer training of the Italian national speed skating short track team. Two years away from the 2014 Sochi Olympics, the coaches were examining how to organize their out-of-season annual training program. According to them, the global culture of short track speed skating causes each of the competing countries to carry out many sessions on the ice in the

Table I Influencing factors on the short track speed skating athlete's performance

\begin{tabular}{|c|c|c|}
\hline Technical factors & Endogenous factors & Exogenous factors \\
\hline Skate & Psychological dispositions & $\begin{array}{l}\text { Values of the coaches, executives, } \\
\text { clubs, federations }\end{array}$ \\
\hline The speed skating boot & Family history & Relevance of selected training stimuli \\
\hline Blade supports & Sporting history & Training partners \\
\hline The blade: & Relationship history & The skating technique taught \\
\hline Metal type & Lifestyle & Physical preparation \\
\hline Rocker angle & Available support & Training results and follow-up \\
\hline Curve angle & Physical qualities & Athlete's nutritional program \\
\hline Sharpening quality & Weight & Quality of sleep \\
\hline Clothing technology & Height & Athlete's social and academic life \\
\hline Suit type & Morphologic type & Psychological performance preparation \\
\hline Textile fiber type & Muscle fiber quality & Opponents \\
\hline Training suit versus competition suit & Physiological dispositions & Perceived post-athletic life situation \\
\hline Hand protection & Motor qualities & \\
\hline Eye protection & Global motor repertoire & \\
\hline Head protection & Short track speed skating & \\
\hline Environment & specific motor skills & \\
\hline Safety, quality, and properties of the ice & Perception of the skating technique's & \\
\hline Protection mattress & specific of the body schematic: & \\
\hline Place of training & Shoulder position & \\
\hline \multirow[t]{13}{*}{ Place of competition } & Hip position & \\
\hline & Knee position & \\
\hline & Ankle position & \\
\hline & Range and efficiency of the skating & \\
\hline & step, cross-over and pivot & \\
\hline & Efficiency of both legs & \\
\hline & Racing experiences: & \\
\hline & Starting technique & \\
\hline & Track knowledge & \\
\hline & Passing strategies & \\
\hline & Energy management & \\
\hline & Strategic positioning & \\
\hline & Trial by error and success & \\
\hline
\end{tabular}

Note: Adapted from Technovation, 25(10), Versluis C, Innovations on thin ice, I I83-1 192, Copyright @ (2005), with permission from Elsevier.' 


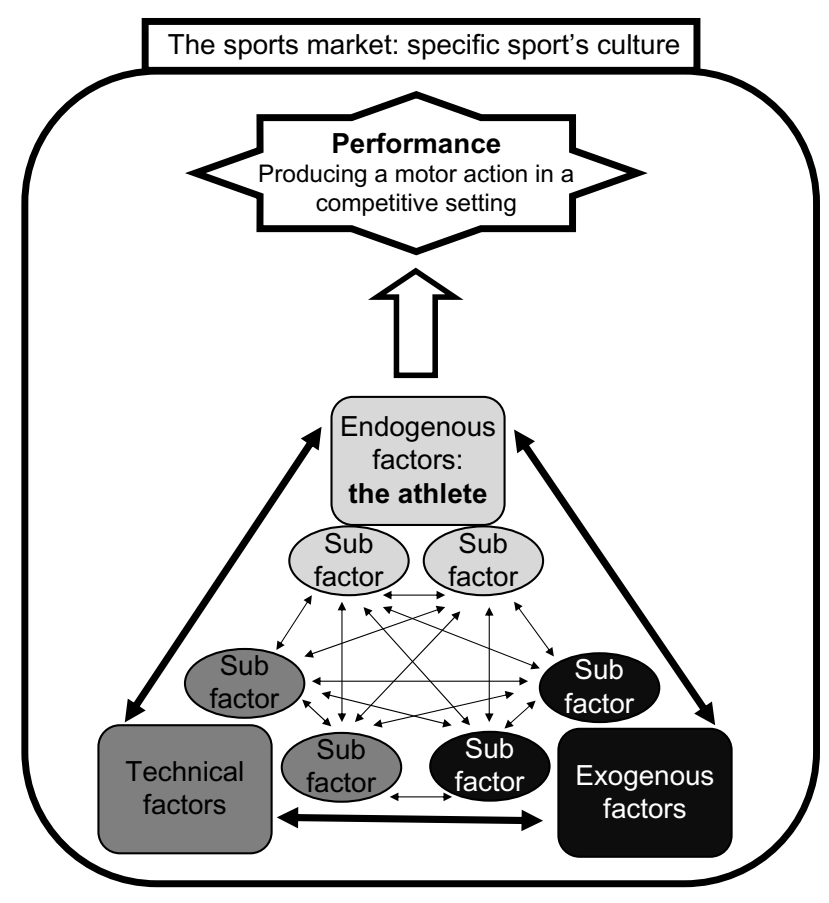

Figure I Interactions between endogenous, exogenous, and technical factors influencing performance on a sports market.

Note: Reprinted from Technovation, 25(10), Versluis C, Innovations on thin ice, I I83-I I92, Copyright @ (2005), with permission from Elsevier.'

course of training. As a matter of fact, two daily sessions on the ice is standard during summer training camp, allowing the athletes to increase the amount of laps in between two seasons of competition.

The increase of the amount of laps in the summer creates significant stress for the adaptation in this specific posture, to such an extent that it can interfere with other training activities. Coaches had noticed that during these increases in training volume, it sometimes became difficult to obtain quality work during off-ice workout sessions, running sessions, core muscle strengthening exercises, as well as sometimes on the ice.

In order to counter this specific problem, the research team decided to make the choice of a training stimulation that potentially had the opposite effect; while the athlete's body adapts to drive the high volume of the specific postures of short track speed skating, what if at the same time, it has to develop a new postural skill: yoga. With this situation, adding yoga sessions fits this need because of its postural nature. This activity, based on knowledge that has been passed down for millennia, offers its practitioners, in a wide range of styles, the execution of a series of postures and breathing exercises. ${ }^{5}$

Research on the benefits of yoga is a growing field. ${ }^{6-9}$ Many sciences and areas of interest are studying its effects. Reviews of literature and narrative synthesis abound, all



Figure 2 Example of back bend.

pointing in the same direction. It appears that yoga has the potential to improve many psychological, physiological, and motor factors, and this, for an eclectic list of conditions: stress and anxiety, ${ }^{10-14}$ many psychiatrics conditions, ${ }^{11,15-19}$ low back pain, ${ }^{15,17,20}$ diabetes and body weight outcomes, ${ }^{21-23}$ cancer, ${ }^{24,25}$ cardiac health, ${ }^{26}$ hypertension, ${ }^{27,28}$ pulmonary conditions ${ }^{28}$ etc. Despite these positive feasible outcomes, research requires more rigorous protocols to seal scientifically valid benefits, including great methodologies for clinical trials and the whole pedagogical issue in the perspective of the intention to treat.

Even though yoga is a popular trend in professional sports, less is known in the scientific literature about its real effects in the matter of strength and conditioning for elite athletes. However, many studies indicate the avenues of exploration: 24 yoga sessions through 8 weeks of Bikram yoga improves strength of the lower limbs; $;{ }^{29}$ measured with kinematic, kinetic, and biomechanical data, Hatha yoga postures showed that they can engender an appreciable range of joint angles through the ankle, knee, and hip, and elicited a qualitative rectus abdominis activity. ${ }^{30}$ The effects on flexibility are also noticed in many positives ways, as well as the delayed onset of muscle soreness in the lower extremity. ${ }^{31,32}$ It has also been shown that yoga "asanas" are effective in teaching musculoskeletal anatomy of the lower limbs. ${ }^{33}$

In this context, coaches decided to add yoga sessions for 8 weeks in a prospective study (which corresponded to 
two training camps separated by a week of rest for the entire team) to the original training plan, to achieve three objectives: 1) to observe whether the addition of the practice of yoga as postural training could improve the efficiency and the athlete's repertoire along with the muscular synergies solicited in the short track speed skating specific technique; 2) to enhance and diversify the motor time-on-task of athletes without changing the prescription of other training stimulations; and 3) to lower the risk of injury during periods with high volumes of training.

To achieve these objectives, the coaches made the hypothesis that adding a training stimulation related to the postural skills, here in the form of yoga customized for short track speed skating, would produce a positive effect in the athlete's physical literacy, and ultimately, this positive effect would be transferred to their specific task. The concept of physical literacy can be defined, as appropriate to each individual's endowment, as a disposition to capitalize on the human embodied capability, wherein the individual has the motivation, confidence, physical competence, knowledge, and understanding to value and take responsibility for maintaining purposeful physical pursuits or activities throughout the life course. ${ }^{34}$

\section{Methods}

\section{Intervention calendar planning}

Initially, the coaches had identified a reality: the volume of all the training stimuli already contained in the training plan could not be increased. In addition, these stimuli already covered the three main motor skills: ${ }^{2}$ locomotion, handling and posture. Locomotion with skating, running, cycling and imitations; handling with bodybuilding; postural skills with stabilization drills and abdominal workout.

Then, in the context of the postural over adaptation in connection with the high volume of laps performed on ice, and with the understanding of the research team on important postural aspects to consider in the practice of short track speed skating, the work of the research team was to make a decision on the nature of the intervention. In the knowledge that comes from yoga, there are a wide variety of styles and a wide repertoire of positions, which lists more than 1,000 positions. In the planning, it was important that the choice of positions would meet the functional needs identified by coaches in relation to the technical requirements of the speed skating technique. The idea was to widen the base of the functional range for segments identified as key elements in the basic position: leg extension, balance and support, hip mobility, and shoulder positioning. In pursuing this objective, 85 positions were identified; these positions can be found in the pictures directory in Supplementary material 1.

During the 8 weeks, the training stimulation's allocation was planned as follows: 16 muscular workout sessions; 14 running or cycling sessions; 22 sessions of stabilization, imitation of skating movements and torso muscular training; and 62 sessions on the ice. Thirty-six yoga sessions were added to this already established plan, as summarized in Table 2. In total, 19 yoga stretch and 17 athletic yoga classes were

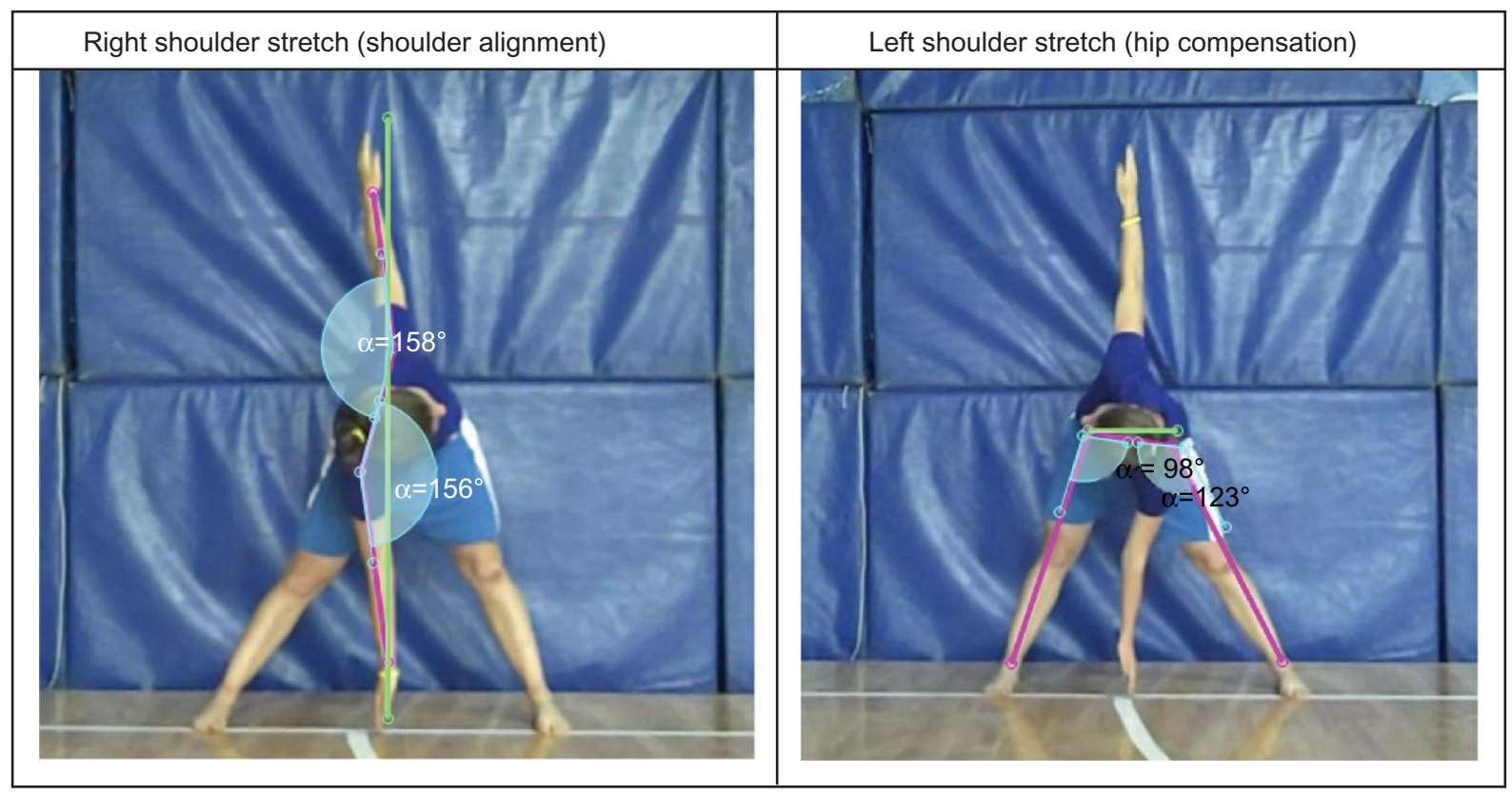

Figure 3 Example of the shoulder stretch. 







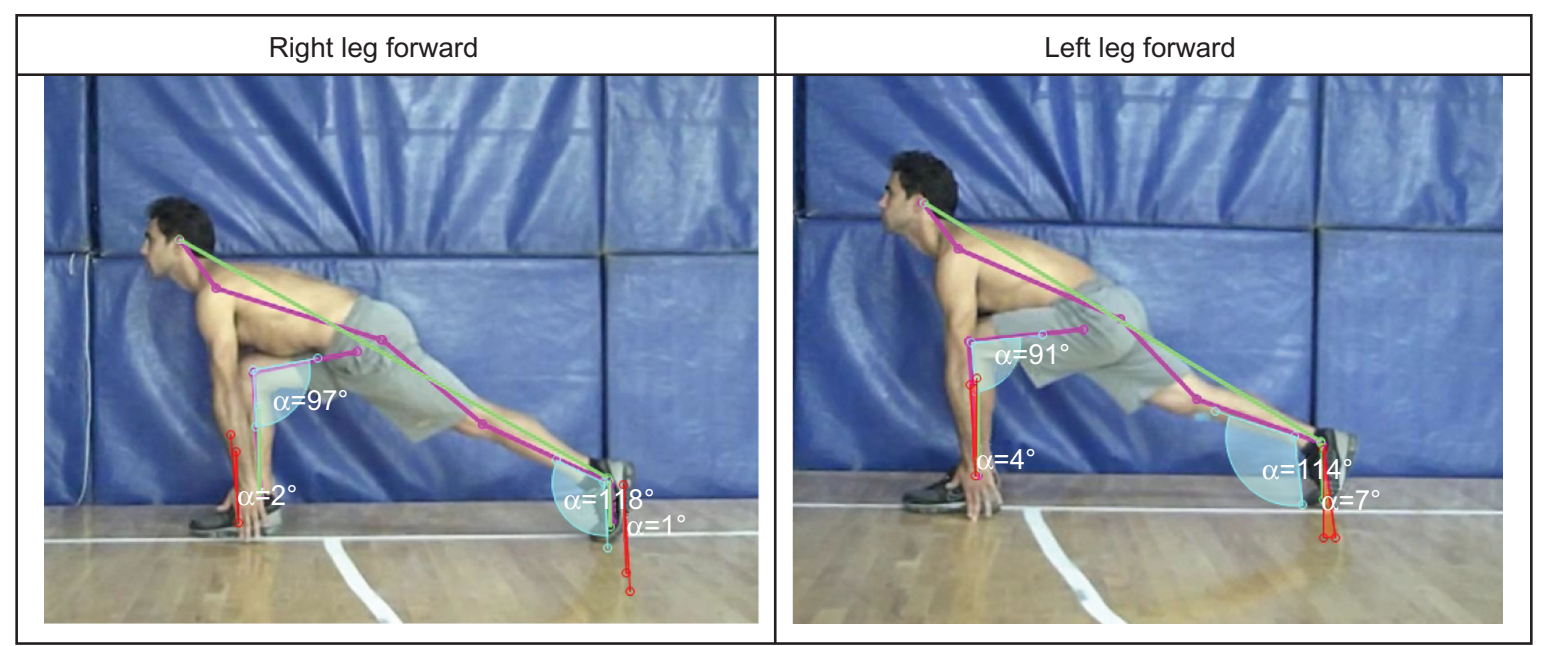

Figure 4 Example of runner's pose.



Figure 5 Two outlier observations in shoulder stretch. 
added. The global planning of the annual training program is available in Supplementary material 2.

\section{Intervention planning}

Once the 85 positions were identified and the general planning was completed, the "decision training" approach was used to plan the learning sequence. ${ }^{35}$ This planning method gives guidelines which place the athletes in an autonomous learning process with the aim to acquire new motor skills. ${ }^{36}$ Out of seven, five of these guidelines were mainly used.

\section{Hard skill task at the beginning of the intervention}

In contrast to a progressive discovery from the simplest positions to the most complex, the athletes were exposed to the challenge that stood before them from the very first session, experimenting with positions with high technical requirements, for instance: yoga position \# 30) the Bikram chair, \# 35) the warrior tree, \# 38) the bonsai, and \# 39) the Bikram arrow.

\section{A varied and random practice}

The intervention structure was prepared in such a way that athletes did not receive the same session twice; the prescription of positions, the rhythm variations, and the order of the sequences were constantly renewed and adjusted to the improvements observed on the field. However, the duration of the sessions was very much the same with an average of 41 minutes.

\section{Modeling}

The modeling approach was adopted throughout the sessions. The yoga coach acted also as a strength and conditioning coach with the team, and had other daily tasks such as supervising warm-ups, bodybuilding, imitations, running training, and abdominal workouts. For theses yoga lessons, he showed, in real time, the postures to take, always with a sense of great execution, with the pleasure of reaching the technical requirements of each posture.

\section{Bandwidth feedback}

In conjunction to the "modeling" approach, the "decision training" approach to feedback commands a limited bandwidth: as long as the coach explicitly broadcasts his instructions, models the actions to reproduce, and can see that the athletes are attempting to do their task properly, he remains silent. Feedback is provided only if: 1) the coach finds that one or more athletes are not properly carrying out the given task or are not on a path to success; or 2) he finds that one or more athletes carry it out perfectly and takes advantage of the situation to reinforce modeling.

\section{Questioning}

If an athlete wanted to discuss the technical aspects of the yoga intervention, instead of giving the answer at hand, the coaching team replied by answering questions on internal feedback perceived by the athlete during the task. Also, at the end of each athletic yoga session, athletes were questioned as to how they had perceived the effort on Borg's scale, ${ }^{37}$ by rating the day's effort from 1 to 10 . The collected data can be found in Supplementary material 3.

\section{Environment, atmosphere, and behaviors during yoga sessions}

The sessions were held in large rooms. Each athlete had an open space to execute positions. Varied background music accompanied the demonstration of the yoga coach. During yoga activities, athletes were asked to remain silent, to focus on having a deep and regular breathing rhythm, and to pay attention to proper technical execution keys and feedback mentioned.

\section{Participants}

Fifteen athletes, seven women and eight men, took part in the 8 weeks of training. This sample is a sample of convenience since these athletes are selected and invited to the national team activities. Average age for women was $21.29 \pm 3.5$ years and their experience on the world cup circuit was on average $4 \pm 2.7$ competitive seasons on the national team. Average age for men was $22.75 \pm 3.28$ years and their experience on the world cup circuit was on average $3 \pm 2.7$ competitive seasons on the national team.

Table 3 Anthropometric measures before the intervention

\begin{tabular}{|c|c|c|c|c|c|c|c|c|c|c|c|c|}
\hline & \multicolumn{2}{|l|}{$\begin{array}{l}\text { Height } \\
(\mathrm{cm})\end{array}$} & \multicolumn{2}{|l|}{$\begin{array}{l}\text { Weight } \\
\text { (kg) }\end{array}$} & \multicolumn{2}{|c|}{ Fat percentage } & \multicolumn{2}{|c|}{$\begin{array}{l}\text { Kilograms of } \\
\text { muscular mass }\end{array}$} & \multicolumn{2}{|c|}{$\begin{array}{l}\text { Muscular mass } \\
\text { body percentage }\end{array}$} & \multicolumn{2}{|c|}{$\begin{array}{l}\text { Body mass } \\
\text { index }\end{array}$} \\
\hline & Ladies & Men & Ladies & Men & Ladies & Men & Ladies & Men & Ladies & Men & Ladies & Men \\
\hline Average & 166.3 & 177.7 & 59 & 75.6 & 20.7 & 10.7 & 24.7 & 38.2 & 41.9 & 50.6 & 21.4 & 23.9 \\
\hline Standard deviation & 3.3 & 8.1 & 4.3 & 7.1 & 2.4 & 2.4 & 1.7 & 3.0 & 1.3 & 1.7 & 23.9 & 1.2 \\
\hline
\end{tabular}


As can be observed in Table 3, despite large standard deviations in size and weight in the women as well as in the men, attention should be paid to the small deviations in terms of body mass index, the body fat ratio as well as the muscle mass ratio to the weight in kilos. These similar ratios could be attributed to shared training stimulations, eating habits, and the annual training program experienced by the team over an extended period.

Fat percentage was measured with a Harpenden skinfold caliper and calculated with the seven folds method (subscapularis, pectoral, triceps, abdominal, pelvic, thigh, and calf). The team doctor administered the test and produced the report which can be found in Supplementary material 4.

\section{Postural measurements}

To measure the evolution of range of motion and body schematic perception, three postural tests were administered before and at the end of the intervention. The protocol of each of the three tests goes at follows: the athlete is asked to hold a specific position for 20 seconds. Execution keys are given explicitly, using anatomic references to guide the athlete. No feedback is given other than the execution keys. The 20-second sequences were filmed with an HD Flip Cam (Cisco Systems, Inc., San Jose, CA, USA). Stills from the clips were made using Sony Vegas software (Sony Creative Software Inc., Middleton, WI, USA), when the athlete showed the best form in the 20 seconds from the execution keys of each test. The angle processing was done using Kinovea software measurement tools (http://www. kinovea.org). This software performs processing angles with a margin of error of 1 degree. All processing of angles was done by a single person who was doubly blinded, both from the content of the research and its outcomes. The markers for the angles were identified manually by the software and some anatomical landmarks, as the anterior superior iliac spines are sometimes hidden by clothing.

\section{Description of the postural tests}

\section{Back bend}

\section{General description}

The back bend consists of, while standing, arms straight and hands joined together, bringing the shoulders as low as possible toward the back. This position shows the range of back and lumbar muscle movement (Figure 2).

\section{Link with short track speed skating}

This functional demonstration of the back is in complete opposition to the muscular synergy associated with the skater's basic position.

\section{Test instructions}

- Position preparation: standing with feet together, hallux and heel touching one another, stretch hands toward the sky, then "glue" them together, while keeping the arms extended with the least possible elbow flexion.

- Moving to the position: pull the chin out toward the sky, and with a controlled movement, push the hips toward the ground by contracting the gluteus without bending the knees, move shoulders toward the back and the ground at the same time as far as possible.

\section{Analyzed angles}

a. Back bend range of motion

- Sky angle: this angle is the result of an angle between a segment parallel to the ground and a segment that passes through the olecranon and left of the center of the palm of the left hand.

- Floor angle: this angle is the result of an angle between a segment perpendicular to the ground and a segment that passes through the lateral malleolus of the left foot and the lateral epicondyle of the left knee.

\section{Angles' relation}

The range of motion is shown in the relationship between the two angles: the greater the sky angle, the lower the score, and a greater amplitude corresponds to a higher score.

\section{Shoulder stretch}

\section{General description}

The shoulder stretch requires a muscular synergy stemming from the hips, a torso twist toward the alignment of the arms and chest, while the feet are placed as wide as possible on the ground (Figure 3).

\section{Link with speed skating}

While the legs are open the shoulder stretch position requires a dissociation of the hips and torso, in a movement that is typical of a skating stride.

\section{Test instructions}

- Place feet wide and parallel, toes and heels of each foot in the same direction.

- Touch the ground with one hand's middle finger, without putting any weight on it. Stretch the opposite hand toward the sky, while trying to form a line, as straight as possible, between the torso and both arms, without bending the knees.

- The athlete must perform this test twice, once on both sides. 


\section{Analyzed angles}

a. Shoulders' alignment

- Sky angle: this angle is the result of an angle between the segment that passes through the left and right acromion and acromion segment that passes through the sky acromion to the head of the radius of the arm outstretched to the sky.

- Floor angle: this angle is the result of an angle between the segment that passes through the right and left acromion and the segment that passes through the acromion to the head of the radius of the arm outstretched to the ground.

\section{Angles' relation}

The closer the result of the addition of the two scores is to 180 (referring to 180 degrees), the more the shoulders and arms tend to form a vertical line.

b. Hips' alignment

- Right hip: this angle is the result of an angle between a segment that passes through both anterior superior iliac spines and a segment that passes through the right anterior superior iliac spine to the malleolus.

- Left hip: this angle is the result of an angle between a segment that passes through both anterior superior iliac spines and a segment that passes through the left anterior superior iliac spine to the malleolus.

\section{Angles' relation}

If the scores of the two measured angles are similar, it indicates that the compensation is small. If there is a big difference, we can identify which hip is compensating, the biggest result indicating the hip that pushes inward.

\section{Runner's position}

\section{General description}

The runner's position requires an important adjustment of the torso's muscle structure, caused mainly by the distance between the supporting leg and the back leg, which is fully extended (Figure 4). It requires a good distribution of balance because the hands cannot be used as a support despite the torso leaning forward.

\section{Link with speed skating}

This position combines many of the muscular qualities required for an efficient skating stride: a strong leg supporting a simultaneously extended "pushed out" leg. If the contraction of the torso muscles is not in harmony with the legs, it becomes difficult to spread out the feet and perform the test requirements.
Test instructions

- Place a supporting foot flat on the floor, and align its knee straight above the supporting ankle, with a 90 degree bend of the leg, placing the thigh at a horizontal angle as much as possible.

- Bring the other foot back as far as possible, while trying to keep the foot at a 90 degree angle compared to the ground, finding balance only with toes. Keep the back leg straight, especially at the knee.

- Then, lower the torso toward the front, until it is possible to touch the ground with the fingertips while keeping the arms straight; fingertips should only barely touch the ground.

- The athlete must perform this test twice, once on both sides.

\section{Analyzed angles}

- Supporting leg to ankle angle: this angle is the result of an angle between a segment from the lateral malleolus to the lateral knee condyle, and a segment perpendicular to the ground.

- Supporting leg to knee angle: this angle is the result of an angle between a segment which passes through the lateral malleolus of the ankle to the lateral epicondyle of the knee and a segment which passes through the lateral epicondyle of the knee to the femoral head.

- Balance foot to ankle angle: this angle is the result of an angle between a segment which passes through the lateral malleolus of the foot toward the metatarsal phalangeal joint of the big toe and a segment perpendicular to the ground.

\section{Angles' relation}

All angles' results show whether the athlete is able to accurately reproduce the commands of the test.

\section{Motor time-on-task}

Motor time-on-task for each planned activity was calculated. Taking into account the nature of each activity, various strategies were used for calculations. Supplementary material 5 brings together most of the elements at the basis of this compilation.

1. Muscular activity: planned actions, number of series, number of repetitions, and their prescribed execution tempo; number of active seconds.

2. Cycling and running activities: running and cycling minutes in real time for non-stop sessions, series, and the number of seconds for the planned volume. Also, the Polar Team ${ }^{2}$ a heart rate monitoring tool (Polar Electro, 
Kempele, Finland), was worn by the 15 athletes in several sessions, allowing collection of time data.

3. Stabilization, imitation, and torso exercise: movements regularly made per second; a precise calculation of exercise time was made.

4. On ice: the number of laps and the prescribed speeds were noted for each training session on the ice. So the calculation was made by compiling these data, while distinguishing laps time made by men and women for this type of activity, since the men have faster paces than women.

5. Yoga: for each yoga session, Polar Team ${ }^{2}$ was worn by the 15 athletes, allowing collection of time data for each session.

\section{Injuries and training activities attendance}

In order to compile the attendance and participation of athletes, a table was made for each day of training, tracking whether the athlete participated in training activities and whether he/she completed some or all of the planned actions. If an absence or non-completion was observed, a note was made explaining the reason for it.

\section{Results \\ Postural tests}

The analysis of the pre-postural and post-postural tests allowed for the identification of significant improvements, with the non-parametric Wilcoxon test, for the following measurements: back bend sky angle $(-11.53 \pm 16.19, P=0.01)$, for the two angles of the shoulder alignment of the right shoulder stretch (sky, left shoulder: 12.07 $\pm 13.34, P=0.005$; floor, right shoulder: $8.07 \pm 12.48, P=0.01$ ), for the floor angle of the right shoulder and for the difference of angles of the hip alignment in the left shoulder stretch (floor, right shoulder: $7.53 \pm 14.45$, $P=0.005$; hips alignment: $-11.00 \pm 12.24, P=0.005$ ), as for all the measurements of angles of the right side of the runner's pose (supporting leg knee-thigh: $4.07 \pm 8.28, P=0.05$; supporting leg knee-ankle: 5.40 $\pm 9.02, P=0.05$; balance leg ankle: $12.00 \pm 16.35, P=0.01$ ), as well as on the left side of the runner's pose (supporting leg knee-thigh: 6.60土6.64, $P=0.005$; supporting leg knee-ankle: 7.60 $\pm 7.88, P=0.005$; balance leg ankle: $14.53 \pm 13.54, P=0.005)$. Table 4 presents all of these results.

\section{Motor time-on-task}

Table 5 shows, per category of training stimulation, the motor time-on-task received by the athletes during the 8 weeks. We can see that the yoga intervention took a total of $30 \%$ of the total time-on-task. Through the 8 weeks, coaches did not notice any change in performance in any of the other activities whatsoever: the compilation of the results for laps on the ice, cycling, and running has shown that athletes managed to accomplish the expected training times despite this major change in their training program. We can then reach the conclusion that adding yoga as a postural training stimulation reached the objectives as it did not interfere with the other training activities and added a significant amount of time spent training.

Furthermore, a quick analysis of the data gathered by Polar Team ${ }^{2}$ has shown that the yoga activities stimulated the athletes' cardiovascular system consistently over $80 \%$ of their maximum heart rate, and even recorded peaks of $91 \%$.

Table 4 Angles results for the postural tests

\begin{tabular}{|c|c|c|c|c|c|}
\hline Test & Angles $\left({ }^{\circ}\right)$ & $\begin{array}{l}\text { Average } \\
\text { difference }\end{array}$ & $\begin{array}{l}\text { Standard } \\
\text { deviation }\end{array}$ & $\mathbf{n}$ & $\begin{array}{l}\text { Wilcoxon } \\
\text { P-value }\end{array}$ \\
\hline \multirow[t]{2}{*}{ Back bend } & Floor angle & 1.87 & 8.07 & 14 & NS: $P>0.05$ \\
\hline & Sky angle & -11.53 & 16.19 & 14 & $P=0.01$ \\
\hline Right shoulder & Sky: left shoulder & 12.07 & 13.34 & 14 & $P=0.005$ \\
\hline \multirow[t]{2}{*}{ stretch } & Floor: right shoulder & 8.07 & 12.48 & 14 & $P=0.01$ \\
\hline & Hips & -2.73 & 12.56 & 15 & NS: $P>0.05$ \\
\hline Left shoulder & Sky: left shoulder & 2.07 & 16.98 & 15 & NS: $P>0.05$ \\
\hline \multirow[t]{2}{*}{ stretch } & Floor: right shoulder & 7.53 & 14.45 & 15 & $P=0.05$ \\
\hline & Hips & -11.00 & 12.24 & 15 & $P=0.005$ \\
\hline Right leg & Supporting leg knee & 4.07 & 8.28 & 14 & $P=0.05$ \\
\hline \multirow[t]{2}{*}{ runner's pose } & Supporting leg ankle-thigh & 5.40 & 9.02 & 14 & $P=0.05$ \\
\hline & Balance leg knee-ankle & 12.00 & 16.35 & 14 & $P=0.01$ \\
\hline Left leg & Supporting leg knee-thigh & 6.60 & 6.64 & 14 & $P=0.005$ \\
\hline \multirow[t]{2}{*}{ runner's pose } & Supporting leg knee-ankle & 7.60 & 7.88 & 15 & $P=0.005$ \\
\hline & Balance leg ankle & 14.53 & 13.54 & 14 & $P=0.005$ \\
\hline
\end{tabular}

Abbreviations: NS, not significant; $n$, number of measures valuable for the Wilcoxon test. 
Table 5 Distribution of the motor time-on-task for all training stimulations

\begin{tabular}{|c|c|c|c|}
\hline Category & $\begin{array}{l}\text { Total time-on-task in } \\
\text { minutes }\end{array}$ & $\begin{array}{l}\text { Motor time-on-task } \\
\text { percentage for the activity }\end{array}$ & $\begin{array}{l}\text { Number of activities by } \\
\text { category over the } 8 \text { weeks }\end{array}$ \\
\hline Yoga & $986 \mathrm{~min}$ & $30 \%$ & 36 \\
\hline Weightlifting session & $500 \mathrm{~min}$ & $15 \%$ & 16 \\
\hline Cycling and running & $\begin{array}{l}\text { Cycling }=560 \mathrm{~min} \\
\text { Running }=174 \mathrm{~min} 30 \mathrm{sec} \\
\text { Agility and speed }=30 \mathrm{~min} \\
\text { Total }=764 \mathrm{~min} 30 \mathrm{sec}\end{array}$ & $24 \%$ & 14 \\
\hline $\begin{array}{l}\text { Stabilization, imitation, } \\
\text { and abs workout }\end{array}$ & $\begin{array}{l}\text { Stabilization }=60 \mathrm{~min} \\
\text { Abs workout }=91 \mathrm{~min} \\
\text { Imitation }=160 \mathrm{~min} \\
\text { Total }=31 \mathrm{I} \mathrm{min}\end{array}$ & $10 \%$ & 22 \\
\hline Ice & $\begin{array}{l}\text { Total } \\
\text { Men }=647 \mathrm{~min} 2 \mathrm{I} \mathrm{sec} \\
\text { Women }=686 \mathrm{~min} 19 \mathrm{sec}\end{array}$ & $21 \%$ & 62 \\
\hline
\end{tabular}

Abbreviations: min, minutes; sec, seconds; abs, abdominal.

\section{Injuries and training activities attendance}

The attendance and activity completion timetables analysis shows us that absences and missed actions always share the same causes: 1) planned absences for external activities (academic tests, attending meetings related to professional activities, exceptional family-related activities); 2) falls during ice activities; and 3 ) infectious diseases (flu, intestinal ailments).

No athlete missed any training due to trauma or discomfort linked to one of the training activities.

\section{Discussion}

In light of these results, we can conclude that the goals set by the coaches for these sessions were met; the postural functionalities were improved and diversified; motor timeon-task was significantly increased due to the yoga sessions and apart from injuries caused by fall, the athletes managed to accomplish all of the planned training activities in accordance with the expected performances.

On the ice, coaches could notice a change in the athlete's perception of their body schematic related to their skating technique. In fact, after the yoga sessions, head coaches felt that the athletes were more capable of dissociating their torso from their hips and thus increased the efficiency of their skating stride. As for the left opening, two outlier observations (Figure 5) were made that changed the statistical significance of the test, and after analyzing the two subjects, we can see that the shoulder variation angle is negative but that the global execution of instructions has improved, particularly concerning hip position, which is a crucial goal of the sessions.

Furthermore, the pre-test could have been globally biased because of an intuitive technical specificity: in the skating technique, the athletes are used to pushing their hips toward the left while keeping their shoulders parallel to the ground. During the pre-test, in order to open their shoulders as much as possible and meet test requirements, the athletes could have reproduced this strategy.

It would also have been relevant to look at the results as a multiple single case study. Considering the results globally has a tendency to make us lose sight of certain obvious improvements. For some of the athletes, the coaches observed an important transfer to their skating technique, but also in the perception of their body schematic; the technical feedback was better assimilated after the sessions. When considering the analysis of the effort perception in long yoga sessions, one factor stands out: despite the increasingly difficult level of the session, on average the athletes kept the same perceived effort as in the very first session, which shows an adaptation to the stimulation.

\section{Conclusion}

Even with a annual training program linked to the global culture of this Olympic sport, it must be said that postural skills were not very present in the organization of training before the introduction of yoga adapted to short track speed skating.

In addition to being linked to the specific skills of the sport, the yoga sessions managed to reach the goals set by the coaches in this high volume out-of-season training period.

This intervention allowed the athletes to create a new functional range, which is usually rare in high level spheres, these individuals often honing the skills specific to their sport. This new functionality was developed without interfering with the other training activities.

Inserting these types of sessions in the annual training program opens a new and promising avenue in many regards: 
the prevention of injuries, the effect of developing new motor skills and their transfer, the effect of developing new postural skills and the effect on the strength of segments, and the effect of the variation of heart rate during the postural intervention on different energy circuits. ${ }^{16,38-40}$

Furthermore, yoga is also recognized for its positive effects on psychological dispositions, which constitutes an important field of expertise in research concerning high performance sport.

In compliance with strict methodological guidelines, as the context of the intervention, the choice of positions, the description of protocols, and the educational strategy, more research would clear up this field of activity and enable coaches to begin to develop postural skills sooner in the long-term development of athletes.

\section{Disclosure}

The authors report no conflicts of interest in this work.

\section{References}

1. Versluis C. Innovations on thin ice. Technovation. 2005;25(10): 1183-1192.

2. Rigal R. Motricité Humaine Fondements et Applications Pédagogiques Tome 2: Développement Moteur. Presses de 1'Université du Québec; 2002. French.

3. Quinn A, Lun V, McCall J, Overend T. Injuries in short track speed skating. Am J Sports Med. 2003;31(4):507-510.

4. de Groot G, Hollander AP, Sargeant AJ, van Ingen Schenau GJ, de Boer RW. Applied physiology of speed skating. J Sports Sci. 1987;5(3):249-259.

5. Patanjali. Yoga-Sutras. Paris: Albin Michel Littérature; 1991.

6. McCall MC. In search of yoga: Research trends in a western medical database. Int J Yoga. 2014;7(1):4.

7. McCall MC, Ward A, Roberts NW, Heneghan C. Overview of Systematic Reviews: Yoga as a Therapeutic Intervention for Adults with Acute and Chronic Health Conditions. Evid Based Complement Alternat Med. 2013;2013:945895.

8. Woodyard C. Exploring the therapeutic effects of yoga and its ability to increase quality of life. Int $J$ Yoga. 2011;4(2):49-54.

9. Field T. Yoga clinical research review. Complement Ther Clin Pract. 2011;17(1):1-8.

10. Chong CS, Tsunaka M, Tsang HW, Chan EP, Cheung WM. Effects of yoga on stress management in healthy adults: A systematic review. Altern Ther Health Med. 2011;17(1):32-38.

11. Sharma M. Yoga as an alternative and complementary approach for stress management a systematic review. J Evid-Based Complementary Altern Med. 2014;19(1):59-67.

12. Khalsa SB, Butzer B, Shorter SM, Reinhardt KM, Cope S. Yoga reduces performance anxiety in adolescent musicians. Altern Ther Health Med. 2013;19(2):34-45.

13. Li AW, Goldsmith CA. The effects of yoga on anxiety and stress. Altern Med Rev. 2012;17(1):21-35.

14. Da Silva TL, Ravindran LN, Ravindran AV. Yoga in the treatment of mood and anxiety disorders: A review. Asian J Psychiatry. 2009;2(1): 6-16.

15. Vancampfort D, Vansteelandt K, Scheewe T, et al. Yoga in schizophrenia: a systematic review of randomised controlled trials. Acta Psychiatr Scand. 2012;126(1):12-20.
16. Hagen I, Nayar US. Yoga for children and young people's mental health and well-being: research review and reflections on the mental health potentials of yoga. Front Psychiatry. 2014;5:35.

17. Balasubramaniam M, Telles S, Doraiswamy PM. Yoga on our minds: a systematic review of yoga for neuropsychiatric disorders. Front Psychiatry. 2012;3:117.

18. Cramer H, Lauche R, Klose P, Langhorst J, Dobos G. Yoga for schizophrenia: a systematic review and meta-analysis. BMC Psychiatry. $2013 ; 13: 32$.

19. Skowronek IB, Mounsey A, Handler L. Clinical Inquiry: can yoga reduce symptoms of anxiety and depression? J Fam Pract. 2014;63(7):398-407.

20. Meyer HB, Katsman A, Sones AC, Auerbach DE, Ames D, Rubin RT. Yoga as an ancillary treatment for neurological and psychiatric disorders: a review. J Neuropsychiatry Clin Neurosci. 2012;24(2):152-164.

21. Rioux JG, Ritenbaugh C. Narrative review of yoga intervention clinical trials including weight-related outcomes. Altern Ther Health Med. 2013;19(3):32-46.

22. Aljasir B, Bryson M, Al-Shehri B. Yoga Practice for the Management of Type II Diabetes Mellitus in Adults: A systematic review. Evid Based Complement Alternat Med. 2010;7(4):399-408.

23. Yang K. A review of yoga programs for four leading risk factors of chronic diseases. Evid Based Complement Alternat Med. 2007;4(4):487-491.

24. Sharma M, Haider T, Knowlden AP. Yoga as an alternative and complementary treatment for cancer: a systematic review. JAltern Complement Med. 2013;19(11):870-875.

25. Sengupta P. Health Impacts of Yoga and Pranayama: A State-of-the-Art Review. Int J Prev Med. 2012;3(7):444-458.

26. Jayasinghe SR. Yoga in cardiac health (a review). Eur J Cardiovasc Prev Rehabil. 2004;11(5):369-375.

27. Hagins M, States R, Selfe T, Innes K. Effectiveness of yoga for hypertension: systematic review and meta-analysis. Evid Based Complement Alternat Med. 2013;2013:649836.

28. Abel AN, Lloyd LK, Williams JS. The effects of regular yoga practice on pulmonary function in healthy individuals: a literature review. J Altern Complement Med. 2013;19(3):185-190.

29. Tracy BL, Hart CE. Bikram yoga training and physical fitness in healthy young adults. $J$ Strength Cond Res. 2013;27(3):822-830.

30. Salem GJ, Yu SS, Wang MY, et al. Physical demand profiles of hatha yoga postures performed by older adults. Evid Based Complement Alternat Med. 2013;2013:165763.

31. Boyle CA, Sayers SP, Jensen BE, Headley SA, Manos TM. The effects of yoga training and a single bout of yoga on delayed onset muscle soreness in the lower extremity. J Strength Cond Res. 2004;18(4): 723-729.

32. Amin DJ, Goodman M. The effects of selected asanas in Iyengar yoga on flexibility: Pilot study. J Bodyw Mov Ther. 2014;18(3): 399-404.

33. Bentley DC, Pang SC. Yoga asanas as an effective form of experiential learning when teaching musculoskeletal anatomy of the lower limb. Anat Sci Educ. 2012;5(5):281-286.

34. Whitehead M. Physical Literacy: Throughout the Lifecourse. Routledge: Taylor and Francis; 2010.

35. Vickers JN. Perception, Cognition, and Decision Training: The Quiet Eye in Action. Champaign, IL: Human Kinetics; 2007.

36. Vickers JN, Livingston LF, Umeris-Bohnert S, Holden D. Decision training: the effects of complex instruction, variable practice and reduced delayed feedback on the acquisition and transfer of a motor skill. J Sports Sci. 1999;17(5):357-367.

37. Borg G. Borg's Perceived Exertion and Pain Scales. Champaign, IL: Human Kinetics; 1998.

38. Sharma M, Rush SE. Mindfulness-based stress reduction as a stress management intervention for healthy individuals: a systematicreview. J Evid Based Complementary Altern Med. 2014;19(4): 271-286. 
39. Bankar MA1, Chaudhari SK, Chaudhari KD. Impact of long term Yoga practice on sleep quality and quality of life in the elderly. $J$ Ayurveda Integr Med. 2013;4(1):28-32.
40. Halpern J, Cohen M, Kennedy G, Reece J, Cahan C, Baharav A. Yoga for improving sleep quality and quality of life for older adults. Altern Ther Health Med. 2014;20(3):37-46.

Open Access Journal of Sports Medicine

\section{Publish your work in this journal}

Open Access Journal of Sports Medicine is an international, peer-reviewed, open access journal publishing original research, reports, reviews and commentaries on all areas of sports medicine. The manuscript management system is completely online and includes a very quick and fair peer-review system.
Visit http://www.dovepress.com/testimonials.php to read real quotes from published authors. 